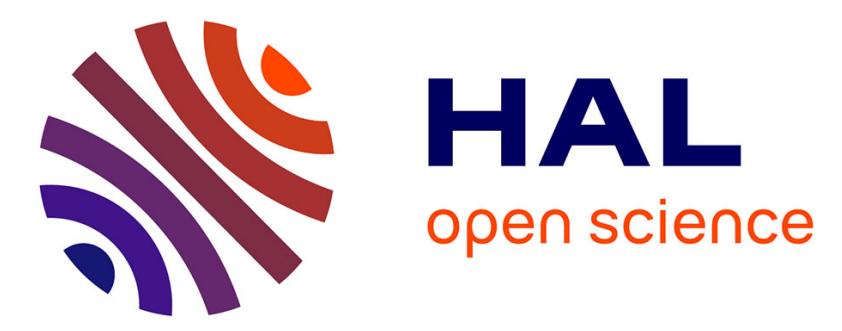

\title{
Les mécanismes de protection des innovations contre l'imitation: un cadre d'analyse générique et un inventaire
}

\author{
Matthieu Mandard
}

\section{- To cite this version:}

Matthieu Mandard. Les mécanismes de protection des innovations contre l'imitation: un cadre d'analyse générique et un inventaire. Gérer et Comprendre. Annales des Mines, 2016, 2016-3 (125), pp.32-40. 10.3917/geco1.125.0032 . halshs-01404247

\section{HAL Id: halshs-01404247 \\ https://shs.hal.science/halshs-01404247}

Submitted on 20 Apr 2021

HAL is a multi-disciplinary open access archive for the deposit and dissemination of scientific research documents, whether they are published or not. The documents may come from teaching and research institutions in France or abroad, or from public or private research centers.
L'archive ouverte pluridisciplinaire HAL, est destinée au dépôt et à la diffusion de documents scientifiques de niveau recherche, publiés ou non, émanant des établissements d'enseignement et de recherche français ou étrangers, des laboratoires publics ou privés. 


\title{
Les mécanismes de protection des innovations contre l'imitation : un cadre d'analyse générique et un inventaire
}

\author{
Matthieu MANDARD \\ Maître de conférences en sciences de gestion \\ Université Rennes 1 \\ Centre de Recherche en Économie et Management (CREM - UMR CNRS 6211) \\ 11 rue Jean Macé - CS 70803 - 35708 RENNES Cedex 7 - FRANCE \\ E-mail : matthieu.mandard@univ-rennes1.fr
}

\section{Résumé}

Comment protéger les innovations contre l'imitation? Si de nombreux mécanismes de protection ont été identifiés ces dernières décennies, ils n'ont pas encore été répertoriés au sein d'un cadre analytique cohérent. C'est l'objectif de cet article, qui propose un cadre d'analyse identifiant six mécanismes génériques de protection des innovations, puis inventorie leur mise en œuvre concrète au niveau inter- et intra-organisationnel, mettant ainsi en évidence une analogie fonctionnelle entre mécanismes de protection à travers les niveaux d'analyse. 
La protection des innovations est un enjeu important pour les économies de la connaissance contemporaines, dans la mesure où les inventeurs échouent fréquemment à profiter de leurs investissements. Il n'est donc pas surprenant que ce sujet ait été particulièrement étudié dans la littérature économique et managériale (James, Leiblein et Lu, 2013). Afin de bénéficier des retombées économiques de leurs innovations, les inventeurs disposent de deux options (Teece, 1986). L'une est de contrôler les actifs complémentaires requis afin de produire et de commercialiser les innovations, tels que des équipements industriels ou des canaux de distribution. L'autre, qui est l'objet de cet article, est de mettre en œuvre des mécanismes de protection des innovations contre l'imitation, également parfois appelés « mécanismes d'appropriations ».

Bien que de nombreux mécanismes de protection des innovations aient été identifiés, tels que le brevet, le secret, l'avance technologique, ou l'existence de pratiques de gestion des ressources humaines spécifiques, jusqu'à présent, ces mécanismes ont généralement été listés plutôt que clairement répertoriés au sein d'un cadre d'analyse cohérent (Hurmelinna-Laukkanen et Puumalainen, 2007 ; James, Leiblein et Lu, 2013). Ceci pose deux problèmes. D'une part, d'un point de vue scientifique, l'absence d'un tel cadre empêche la délimitation de l'étude des mécanismes de protection en tant que champ de recherche, et les zones qui nécessitent de nouvelles investigations ne peuvent être précisément identifiées. D'autre part, d'un point de vue managérial, l'absence de ce cadre explicatif est susceptible d'entraver la transmission des connaissances sur le sujet, augmentant ainsi le risque que les inventeurs ne soient pas au fait des mécanismes de protection existants et subissent des détournements d'innovations qui auraient pu être évités.

L'objectif de cet article est donc de traiter cette question, en proposant un cadre d'analyse générique qui identifie six mécanismes génériques de protection des innovations contre l'imitation - c'est-àdire six catégories principales de mécanismes de protection -, puis en inventoriant la mise en œuvre concrète de ces six mécanismes génériques au niveau inter- comme intra-organisationnel, mettant ainsi en évidence une analogie fonctionnelle entre mécanismes de protection à travers les niveaux 
d'analyse. Concernant cet inventaire, il convient de noter que l'objectif de l'article n'est pas d'effectuer une revue de la littérature qui recenserait systématiquement les antécédents et les conséquences du recours aux différents mécanismes de protection, mais bien de répertorier les différents mécanismes existants et d'en offrir une présentation succincte.

L'article est structuré de la manière suivante. Nous commençons par présenter le cadre d'analyse générique proposé, qui met en évidence six mécanismes génériques de protection des innovations. À partir de ce cadre, nous inventorions ensuite les manifestations concrètes de ces six mécanismes génériques au niveau inter- comme intra-organisationnel. Nous concluons finalement en discutant les implications de cette analyse, à la fois d'un point de vue scientifique et managérial.

\section{Les mécanismes de protection des innovations contre l'imitation : un cadre d'analyse générique}

Ainsi que souligné en introduction, si la littérature portant sur la protection des innovations a crû de manière considérable au cours de ces dernières décennies, un cadre analytique qui recense et articule les différents mécanismes afférents fait toujours défaut. Afin de tenter de pallier ce problème, nous proposons ci-dessous un cadre d'analyse générique qui met en évidence ce que nous appelons six mécanismes génériques de protection, c'est-à-dire six principales catégories de mécanismes de protection des innovations contre l'imitation (Tableau 1). 
Tableau 1 - Les mécanismes de protection des innovations contre l'imitation : un cadre d'analyse générique ${ }^{1}$

\section{Locus de protection}

\begin{tabular}{llccc} 
& Actif & Relation & Acteur \\
\hline $\begin{array}{l}\text { Type de } \\
\text { mécanisme de } \\
\text { protection }\end{array}$ & $\begin{array}{l}\text { Relatif aux } \\
\text { comportements }\end{array}$ & $\begin{array}{c}\text { Droits de propriété } \\
\text { intellectuelle }\end{array}$ & $\begin{array}{c}\text { Établissement de } \\
\text { relations de long- } \\
\text { terme }\end{array}$ & $\begin{array}{c}\text { Normes de } \\
\text { propriété } \\
\text { intellectuelle }\end{array}$ \\
& $\begin{array}{l}\text { Relatif aux } \\
\text { compétences }\end{array}$ & Secret & $\begin{array}{c}\text { Réduction de la } \\
\text { focale des relations }\end{array}$ & $\begin{array}{c}\text { Asymétrie de } \\
\text { compétences }\end{array}$ \\
\hline
\end{tabular}

Bâti de manière inductive à partir de l'étude des divers mécanismes de protection des innovations existants, ce cadre se compose de deux dimensions. Premièrement, nous proposons d'opérer une distinction entre deux types de mécanismes. Nous postulons que les mécanismes de protection sont employés soit pour prévenir un risque comportemental, soit pour prévenir un risque en matière de compétences; alors que (1) les mécanismes relatifs aux comportements sont employés pour prévenir la réalisation effective d'une imitation, (2) les mécanismes relatifs aux compétences agissent en amont, en empêchant l'obtention de la capacité à réaliser une imitation. Dans le premier cas, les imitateurs peuvent être techniquement capables de réaliser une imitation, mais les mécanismes relatifs aux comportements interdisent la mise en œuvre de cette aptitude. Dans le second, la réalisation effective d'une imitation ne nécessite pas d'être empêchée, dans la mesure où les mécanismes relatifs aux compétences auront préalablement privé les imitateurs potentiels de toute aptitude à imiter.

Deuxièmement, nous proposons également de distinguer trois loci de protection - nous appelons « locus de protection » une cible spécifique d'un mécanisme de protection des innovations. Si l'on

1 Ce cadre d'analyse générique est une version remaniée et étendue d'un cadre d'analyse initialement élaboré pour l'étude plus particulière des alliances de R\&D (Mandard, 2013). 
adopte une perspective en termes de réseau social, tout acteur économique est encastré dans des réseaux de relations composés d'un ensemble d'autres acteurs afin d'échanger divers actifs (Borgatti et Halgin, 2011). En lien direct avec ce constat, on peut noter que tout mécanisme de protection agit sur l'une ou l'autre de ces dimensions fondamentales des réseaux : (1) certains mécanismes ont pour but de prévenir l'imitation d'un actif donné, (2) certains mécanismes visent à prévenir l'imitation au sein d'une relation donnée, et (3) certains mécanismes ont pour objectif de prévenir l'imitation par un acteur donné. Cette observation est cohérente avec des travaux précédents, qui ont distingué différentes cibles des mécanismes de protection (Li, Eden, Hitt et Ireland, 2008).

Ces deux distinctions permettent finalement d'aboutir à la mise en évidence de six mécanismes génériques de protection des innovations contre l'imitation. Ainsi que nous allons le voir dans la partie suivante, ces six mécanismes génériques permettent d'inventorier l'ensemble des pratiques de protection des innovations, que ce soit au niveau inter- ou au niveau intra-organisationnel.

\section{Les mécanismes de protection des innovations contre l'imitation : un inventaire}

Il s'agit dans cette section de montrer comment les six mécanismes génériques précédemment mis en évidence sont concrètement mis en œuvre. Pour chaque mécanisme générique, nous détaillons successivement son principe de fonctionnement et ses manifestations concrètes et ce, au niveau inter- comme intra-organisationnel. Cet inventaire est résumé dans le Tableau 2. 
Tableau 2 - Les mécanismes de protection des innovations contre l'imitation : un inventaire

\begin{tabular}{|c|c|c|c|}
\hline $\begin{array}{l}\text { Mécanisme } \\
\text { générique de } \\
\text { protection }\end{array}$ & Principe de fonctionnement & $\begin{array}{c}\text { Mise en ouvre au niveau inter- } \\
\text { organisationnel }\end{array}$ & $\begin{array}{c}\text { Mise en ouvre au niveau intra- } \\
\text { organisationnel }\end{array}$ \\
\hline $\begin{array}{l}\text { Droits de propriété } \\
\text { intellectuelle }\end{array}$ & $\begin{array}{l}\text { Prévenir les comportements d'imitation } \\
\text { relatifs à un actif donné au travers de } \\
\text { l'acquisition du droit d'empêcher autrui de } \\
\text { reproduire cet actif }\end{array}$ & $\begin{array}{l}\text { Acquisition de droits de propriété } \\
\text { intellectuelle afin d'obtenir une } \\
\text { protection envers les concurrents }\end{array}$ & $\begin{array}{l}\text { Acquisition de droits de propriété } \\
\text { intellectuelle afin d'obtenir une } \\
\text { protection envers les employés }\end{array}$ \\
\hline Secret & $\begin{array}{c}\text { Prévenir l'acquisition de compétences en } \\
\text { matière d'imitation relatives à un actif donné } \\
\text { au travers de la rétention des connaissances } \\
\text { pertinentes }\end{array}$ & Secret industriel & $\begin{array}{l}\text { Procédures de détention du secret } \\
\text { industriel }\end{array}$ \\
\hline $\begin{array}{l}\text { Établissement de } \\
\text { relations de long- } \\
\text { terme }\end{array}$ & $\begin{array}{l}\text { Prévenir les comportements d'imitation au } \\
\text { sein d'une relation donnée au travers de la } \\
\text { mise en œuvre d'échanges sur le long terme }\end{array}$ & $\begin{array}{c}\text { Formellement : alliances } \\
\text { hiérarchiques ; } \\
\text { Informellement }: \text { investissement dans } \\
\text { des actifs spécifiques }\end{array}$ & $\begin{array}{l}\text { Formellement : accords de non- } \\
\text { concurrence ; } \\
\text { Informellement : mécanismes de } \\
\text { restriction de la mobilité } \\
\text { professionnelle }\end{array}$ \\
\hline $\begin{array}{l}\text { Réduction de la } \\
\text { focale des relations }\end{array}$ & $\begin{array}{c}\text { Prévenir l'acquisition de compétences en } \\
\text { matière d'imitation au sein d'une relation } \\
\text { donnée au travers de la restriction des } \\
\text { échanges }\end{array}$ & Réduction de la focale des alliances & Procédures d'accès au secret industriel \\
\hline $\begin{array}{l}\text { Normes de propriété } \\
\text { intellectuelle }\end{array}$ & $\begin{array}{c}\text { Prévenir les comportements d'imitation par } \\
\text { un acteur donné au travers de l'exercice de } \\
\text { contraintes informelles sur son } \\
\text { comportement }\end{array}$ & $\begin{array}{l}\text { Mise en œuvre de normes de propriété } \\
\text { intellectuelle entre les organisations }\end{array}$ & $\begin{array}{l}\text { Mise en œuvre de normes de propriété } \\
\text { intellectuelle au sein des organisations }\end{array}$ \\
\hline $\begin{array}{l}\text { Asymétrie de } \\
\text { compétences }\end{array}$ & $\begin{array}{c}\text { Prévenir l'acquisition de compétences en } \\
\text { matière d'imitation par un acteur donné au } \\
\text { travers de l'établissement d'un écart de } \\
\text { compétences }\end{array}$ & Avance technologique & Modularité \\
\hline
\end{tabular}




\section{Les droits de propriété intellectuelle}

Le principe de fonctionnement des droits de propriété intellectuelle est de prévenir les comportements d'imitation relatifs à un actif donné au travers de l'acquisition du droit d'empêcher autrui de reproduire cet actif. Si les droits de propriété intellectuelle sont généralement acquis par les organisations afin de se protéger des organisations concurrentes, elles les acquièrent parfois de manière préventive afin de se protéger d'éventuelles pratiques d'imitation par leurs employés.

\section{Les droits de propriété intellectuelle envers les concurrents}

Les droits de propriété intellectuelle tels que le brevet ou la marque déposée peuvent être définis d'une manière générale comme un ensemble de droits exclusifs accordés à un inventeur pour une invention et pour une période de temps données, empêchant ainsi temporairement autrui de reproduire cette invention. Le brevet est l'un des mécanismes de protection les plus connus, qui est employé dans le monde entier afin de protéger les innovations contre les concurrents (Somaya, 2012).

Bien qu'extrêmement populaires, les droits de propriété intellectuelle tels que le brevet souffrent cependant d'un certain nombre de limites dans leur capacité à protéger les innovations (James, Leiblein et Lu, 2013). D'une part, ils sont coûteux à acquérir et à défendre. D'autre part, ils ne garantissent pas entièrement l'appropriation des retombées économiques des innovations, dans la mesure où leur efficacité varie en fonction des contextes législatifs et industriels. Ce sont les raisons pour lesquelles les organisations telles que les Petites et Moyennes Entreprises (PME) ont souvent besoin de recourir à des mécanismes de protection alternatifs afin de protéger leurs innovations, que nous allons passer en revue dans la suite.

\section{Les droits de propriété intellectuelle envers les employés}

Outre la protection contre l'imitation au niveau inter-organisationnel, qui constitue leur objectif 
premier, les droits de propriété intellectuelle sont parfois acquis préventivement afin d'offrir une protection au niveau intra-organisationnel. Les droits de propriété intellectuelle peuvent en effet être obtenus afin de prévenir les risques associés à une mobilité des employés lorsque ces derniers sont susceptibles de quitter leur employeur afin d'exploiter des inventions pour leur propre compte (Kim et Marschke, 2005).

Ainsi par exemple, une étude portant sur des entreprises françaises innovantes a récemment montré que le risque de départ de leurs chercheurs augmente la propension des entreprises à breveter leurs innovations (Gallié et Legros, 2012). De manière additionnelle, des travaux ont également montré que la réputation de fermeté des organisations en matière de défense de leurs droits de propriété intellectuelle renforce la protection offerte par ces droits au niveau intra-organisationnel en diminuant les fuites de connaissances associés à la mobilité des inventeurs (Ganco, Ziedonis et Agarwal, 2015).

\section{Le secret}

Alors que le principe de fonctionnement des droits de propriété intellectuelle est de prévenir les comportements d'imitation, le principe de fonctionnement du secret est de prévenir l'acquisition de compétences en matière d'imitation relatives à un actif donné au travers de la rétention des connaissances pertinentes. Ce mécanisme générique peut être mis en œuvre de deux manières : au travers du secret industriel au niveau inter-organisationnel, et au travers des procédures de détention du secret industriel au niveau intra-organisationnel.

\section{Le secret industriel et les accords de non-divulgation}

De manière générale, un secret industriel est un ensemble de connaissances intentionnellement dissimulé par une organisation afin que celles-ci ne soient acquises par d'autres organisations (Hannah, 2005). De même que les droits de propriété intellectuelle, il s'agit d'un mécanisme de 
protection populaire au niveau inter-organisationnel (James, Leiblein et Lu, 2013). Trois critères définissent un secret industriel (Hannah, 2005) : (1) le secret industriel doit contenir des connaissances ; (2) le secret industriel doit présenter une valeur pour l'organisation qui le possède, et cette valeur doit provenir du caractère secret des connaissances détenues ; (3) l'organisation doit faire des efforts raisonnables afin de protéger le secret industriel - il s'agit ici de mettre en œuvre des mécanismes internes tels que ceux décrits dans la suite de l'article. Le secret industriel est particulièrement apprécié par les PME, dans la mesure où il est moins coûteux à mettre en œuvre que les droits de propriété intellectuelle (Leiponen et Byma, 2009). On peut noter que, dans des industries telles que celles des semi-conducteurs ou des logiciels informatiques, le secret industriel contenu dans des artefacts peut en outre être protégé par l'interdiction de la rétro-ingénierie (Samuelson et Scotchmer, 2002).

\section{Les procédures de détention du secret industriel}

La prévention de l'acquisition de compétences relatives à un actif donné au travers de la rétention des connaissances pertinentes peut également être mise en œuvre au sein des organisations, au travers de l'établissement de procédures de détentions du secret industriel. Ces procédures visent à préserver la confidentialité de certaines connaissances en édictant des règles qui définissent les marges de manœuvre des employés qui en disposent (Hannah, 2005). Elles sont communément mises en œuvre de deux manières. D'une part, les accords de non-divulgation peuvent formellement interdire la diffusion des connaissances sensibles de la part des employés qui y ont accès. D'autre part, les procédures relatives à la non-documentation des connaissances considérées comme précieuses peuvent également permettre de limiter leur diffusion (Keupp, Beckenbauer et Gassmann, 2009). De telles procédures de détention du secret industriel sont par exemple mises en œuvre par l'entreprise KFC auprès des employés qui ont accès à des recettes dont elle souhaite préserver la confidentialité (Hannah, 2005). 
Il est intéressant de noter que, en droit états-uniens, lorsque des employés sont tenus de garder des connaissances secrètes, les tribunaux peuvent délivrer une injonction pour divulgation inévitable si ces employés entreprennent de quitter leur entreprise pour des concurrents (Liebman, Kahnke et Bundy, 2005). Ces injonctions interdisent aux employés de travailler pour les concurrents ; elles sont délivrées lorsque les employés seront inévitablement amenés à dévoiler des informations confidentielles en réalisant un travail similaire pour des concurrents - par exemple, en accentuant les efforts de recherche dans une direction qu'ils savent fructueuse car elle aura été préalablement expérimentée dans leur entreprise d'origine -, même si aucune connaissance sensible n'a été divulguée en tant que telle.

\section{L'établissement de relations de long-terme}

Le principe de fonctionnement de l'établissement de relations de long-terme est de prévenir les comportements d'imitation au sein d'une relation donnée au travers de la mise en œuvre d'échanges sur le long terme. Ainsi que nous allons le voir, que ce soit au niveau inter- ou au niveau intraorganisationnel, les relations de long-terme en tant que mécanisme de protection peuvent être mises en œuvre formellement ou informellement soit, respectivement, au travers de contrats ou de relations solidement établies.

\section{Les alliances hiérarchiques et l'investissement dans des actifs spécifiques}

Positionné dans la perspective de la théorie des coûts de transaction, un ensemble de travaux a mis en évidence un lien entre les risques de détournement d'innovations et la structure contractuelle des relations inter-organisationnelles. Les recherches ont ainsi montré que les relations interorganisationnelles qui comportent des risques d'opportunisme adoptent plus volontiers des structures d'alliances hiérarchiques au travers de la création d'une coentreprise (Hagedoorn, Cloodt et Van Kranenburg, 2005 ; Oxley et Sampson, 2004). Le recours à une structure telle qu'une 
coentreprise permet en effet de limiter ces risques pour trois raisons (Oxley, 1999): (1) la propriété commune de la coentreprise réduit l'incitation à l'opportunisme et encourage les comportements collaboratifs ; (2) les futurs profits issus de la coentreprise diminuent les bénéfice associés à un comportement opportuniste ponctuel ; (3) la participation commune à la supervision des activités de la coentreprise favorise une surveillance mutuelle qui restreint les possibilités de détournement d'innovations. À titre d'exemple, ces raisons expliquent en partie pourquoi la collaboration en matière de Recherche et Développement (R\&D) nouée en 2000 entre les entreprises Nikon et Essilor a pris la forme d'une coentreprise.

Au niveau inter-organisationnel, les risques en matière d'imitation peuvent également être réduits de manière informelle au travers d'investissements dans des actifs spécifiques. Des organisations qui collaborent fréquemment sont susceptibles d'avoir développé des normes collaboratives ou d'avoir investi dans des équipements dédiés à leur relation (par exemple, dans des systèmes d'information ou dans des machines) qui leurs permettent de mettre en place des échanges efficients et profitables. Ces actifs spécifiques constituent de fait un frein à l'adoption de comportements d'imitation dans la mesure où, de manière similaire à la mise en œuvre formelle de relations de long-terme, les futurs profits issus des échanges à venir atténuent les risques d'opportunisme en réduisant les incitations des partenaires à agir de manière opportuniste pour un bénéfice ponctuel (Kale, Singh et Perlmutter, 2000 ; Li, Eden, Hitt et Ireland, 2008).

\section{Les accords de non-concurrence et les mécanismes de restriction de la mobilité professionnelle}

De manière analogue au niveau inter-organisationnel, au sein des organisations, des relations de long-terme avec les employés peuvent être mises en place de manière formelle : au travers d'accords de non-concurrence, les employés peuvent se retrouver liés à une organisation donnée et ainsi empêchés pour plusieurs années de rejoindre des concurrents, ce qui offre de fait une protection pour les innovations de leur organisation d'appartenance (Marx, 2011). Il n'est donc pas surprenant 
que plus l'efficacité juridique des accords de non-concurrence est grande, et plus les entreprises tendent à s'engager dans des projets de recherche et de développement risqués, dans la mesure où elles seront plus susceptible d'en récolter les fruits. Ceci a par exemple été montré dans le cas des entreprises états-uniennes (Conti, 2014).

$\mathrm{Au}$ sein des organisations, des relations de long-terme peuvent également être développées informellement au travers de la mise en œuvre de mécanismes de restriction de la mobilité professionnelle tels que des salaires élevés, des plans de carrière attractifs, ou la mise en place d'un environnement de travail stimulant (Delerue et Lejeune, 2010 ; Liebeskind, 1997). En créant les conditions d'une relation profitable entre un employeur et ses employés et en réduisant ainsi l'incitation de ces derniers à l'adoption d'un comportement opportuniste ponctuel, ces pratiques favorisent en effet la rétention des employés innovants et la protection des innovations des organisations. Ce sont notamment des pratiques adoptées par les PME du secteur des biotechnologies, dont les activités reposent avant tout sur le travail effectué par leur personnel scientifique (Delerue et Lejeune, 2010).

\section{La réduction de la focale des relations}

Alors que le principe de fonctionnement de l'établissement de relations de long-terme est de prévenir les comportements d'imitation, la réduction de la focale des relations a pour objet de prévenir l'acquisition de compétences en matière d'imitation au sein d'une relation donnée au travers de la restriction des échanges. À la différence du secret qui vise spécifiquement à taire les compétences relatives à une innovation particulière, la réduction de la focale des relations vise plus généralement à diminuer les points de contact entre l'inventeur et les imitateurs potentiels. Ce mécanisme générique peut être mis en œuvre de deux manières : au travers de la réduction de la focale des alliances au niveau inter-organisationnel, et au travers des procédures d'accès au secret industriel au niveau intra-organisationnel. 


\section{La réduction de la focale des alliances}

Au niveau inter-organisationnel, la réduction de la focale des alliances entre des organisations vise à restreindre les échanges avec les interlocuteurs et prévenir ainsi l'acquisition de compétences (Oxley et Sampson, 2004). Il s'agit d'un mécanisme communément mis en œuvre au sein des alliances qui comportent des risques en matière technologique. Une étude portant sur les collaborations internationales d'entreprises appartenant au secteur des télécommunications et de l'électronique a ainsi montré que plus ces échanges sont risqués et plus la focale des alliances est susceptible d'être réduite (Oxley et Sampson, 2004).

Afin de réduire la focale des alliances, les organisations disposent de deux options. Premièrement, elles peuvent limiter le nombre d'activités en jeu dans les échanges. Par exemple, lorsque des organisations collaborent avec des concurrents dans le cadre de projets d'innovation, elles sont plus susceptibles d'exclure les activités de production et de commercialisation de leurs échanges, ceci afin de réduire la capacité des concurrents à exploiter les innovations générées pour leur propre profit (Li, Eden, Hitt et Ireland, 2008 ; Oxley et Sampson, 2004). Deuxièmement, les organisations peuvent également limiter le contenu des activités concernées par la collaboration. Ainsi, lorsqu'elles collaborent avec des concurrents, les organisations sont plus susceptibles de réduire le nombre d'employés ou la quantité de produits ou de marchés impliqués dans l'échange afin de contenir les transferts de connaissances vers ses partenaires (Hamel, 1991).

\section{Les procédures d'accès au secret industriel}

De manière analogue au niveau inter-organisationnel, au sein des organisations, le risque d'acquisition de compétences en matière d'imitation par les employés peut être réduit au travers de la restriction des échanges que leur organisation d'appartenance réalise avec eux. Ceci est communément mis en œuvre via l'établissement de procédures d'accès au secret industriel, qui sont 
de deux ordres (Hannah, 2005). D'une part, ces procédures peuvent viser à limiter l'accès des employés à certaines parties des installations des organisations. Par exemple, l'accès aux laboratoires peut être réservé aux chercheurs qui en sont membres. Une telle pratique est en outre facilitée lorsque les bâtiments sont isolés géographiquement du reste de l'organisation (Liebeskind, 1997). D'autre part, ces procédures peuvent également viser à limiter l'accès des employés à des documents physiques ou électroniques ainsi que leur droit à les utiliser et à les copier. Ces deux types de pratiques sont mis fréquemment mis en œuvre dans les centres de R\&D tels que les technocentres de Renault ou d'Orange.

\section{Les normes de propriété intellectuelle}

Le principe de fonctionnement des normes de propriété intellectuelle est de prévenir les comportements d'imitation par un acteur donné au travers de l'exercice de contraintes informelles sur son comportement. De même que dans le cas des droits de propriété intellectuelle, ces normes peuvent être à la fois mises en œuvre entre les organisations ou en leur sein.

\section{Les normes de propriété intellectuelle entre les organisations}

Les normes de propriété intellectuelle sont des règles sociales qui régulent la propriété intellectuelle au sein d'un réseau d'acteurs et qui imposent des sanctions sur ceux qui la violent (Fauchart et Von Hippel, 2008 ; Oliar et Sprigman, 2008). De telles normes ne sont pas associés à des règles formelles ; suivant une logique en termes de théorie des jeux, elles sont basées sur une évaluation du rapport entre les profits issus des collaborations à venir avec les autres membres d'un réseau et les gains issus du détournement ponctuel d'une innovation. Lorsque les membres d'un réseau sont interdépendants et densément connectés, les sanctions collectives sont ainsi connues pour dissuader les comportements opportunistes, qui deviennent non-rentables.

Les normes de propriété intellectuelle sont fréquemment employées dans les industries dans 
lesquelles les inventions ne peuvent être protégées par les droits de propriété intellectuelle, telles que dans la haute gastronomie (Di Stefano, King et Verona, 2014 ; Fauchart et Von Hippel, 2008), ou dans l'industrie du spectacle comique (Oliar et Sprigman, 2008). Dans ces industries où tous les acteurs sont largement interconnectés, les imitateurs courent en effet le risque d'être exclus de leur communauté et d'être privés d'échanges ultérieurs. Au-delà de ces industries particulières, des recherches ont récemment montré que des normes de propriété intellectuelle peuvent également être imposées dans les industries de haute technologie, protégeant ainsi les innovations techniques (Haeussler, 2011 ; Hallen, Katila et Rosenberger, 2014).

\section{Les normes de propriété intellectuelle au sein des organisations}

De manière analogue, les normes de propriété intellectuelle peuvent également être mises en œuvre au niveau intra-organisationnel via l'établissement de règles sociales qui définissent et imposent des normes de propriété intellectuelle parmi les employés. Lorsque des employés entretiennent des liens sociaux et partagent un attachement commun à la destinée de leur organisation d'appartenance, un contrôle social est en effet parfois exercé sur leur comportement qui freine l'opportunisme (Costas et Grey, 2014 ; Lazega, 2000). Le principe de fonctionnement est simple : de même qu'au niveau inter-organisationnel, les employés coupables d'imitation courent le risque d'être exclus de leur communauté, ce qui rend l'opportunisme coûteux. Ici également, plus les individus sont interdépendants et interconnectés, et plus les comportements opportunistes sont découragés.

Ainsi, en France, le cas de l'industrie sidérurgique du Creusot au cours des 19 ème et 20ème est emblématique de ce type de pratique. La plupart des habitants de la ville étaient employés dans l'entreprise Schneider, ce qui induisait un fort contrôle sur leurs comportements dans la mesure où vie professionnelle et vie privée étaient intimement liées. Plus récemment, en Chine, une recherche a de nouveau montré que de forts liens sociaux entre employés associés à un fort attachement à leur employeur sont susceptibles d'offrir une réelle protection pour les innovations de leur entreprise 
(Keupp, Beckenbauer et Gassmann, 2009).

\section{L'asymétrie de compétences}

$\mathrm{Au}$ contraire des normes de propriété intellectuelle qui visent à prévenir les comportements d'imitation, le principe de fonctionnement de l'asymétrie de compétences est de prévenir l'acquisition de compétences en matière d'imitation par un acteur donné au travers de l'établissement d'un écart de compétences. Ce mécanisme générique peut être mis en œuvre de deux manières : au travers de l'avance technologique au niveau inter-organisationnel, et au travers de la modularité au niveau intra-organisationnel.

\section{L'avance technologique}

L'asymétrie de compétences peut tout d'abord être mise en œuvre au niveau inter-organisationnel au travers de l'établissement d'une avance technologique, qui correspond à un délai entre la mise sur le marché d'une innovation par une organisation et l'imitation de cette innovation par un concurrent. Ce délai permet à l'organisation en question de retirer temporairement un bénéfice économique de son innovation le temps que le concurrent devienne compétent pour réaliser des imitations. Tout l'enjeu consiste ici à mettre sur le marché de nouveaux produits de manière régulière afin de constamment conserver cette avance. Ce mécanisme de protection est particulièrement employé par les PME innovantes, qui ne disposent pas toujours des ressources pour acquérir des droits de propriété intellectuelle et les défendre (Leiponen et Byma, 2009).

Il convient de noter que la nature des connaissances a un effet modérateur sur l'efficacité de l'avance technologique, dans la mesure où plus les connaissances sont complexes et plus cette avance est susceptible d'être durable (McEvily et Chakravarthy, 2002). De la même manière, l'intensité compétitive d'une industrie modère également l'efficacité de l'avance technologique en tant que mécanisme de protection: plus la concurrence est grande et plus cette avance se trouve vite 
comblée (James, Leiblein et Lu, 2013). ${ }^{2}$

\section{La modularité}

$\mathrm{Au}$ niveau intra-organisationnel, la prévention de l'acquisition de compétences en matière d'imitation par un acteur donné au travers de l'établissement d'un écart de compétences peut également se faire, ceci au travers de la mise en œuvre d'une démarche de modularité. La modularité se définit comme le partitionnement des connaissances techniques relatives à un produit ou à un processus (Baldwin et Henkel, 2014). Ce partitionnement permet aux organisations de diviser entre les employés les connaissances jugées sensibles, ce qui les rend individuellement incapable de reproduire entièrement une innovation. De manière analogue à l'avance technologique, plus une innovation est complexe, et plus il est aisé de mettre en œuvre une démarche de modularité. Ce mécanisme est notamment employé dans l'industrie chimique, dans laquelle l'élaboration des produits nécessite souvent de nombreuses étapes qu'il est possible de cloisonner afin de préserver la confidentialité du processus global (Keupp, Beckenbauer et Gassmann, 2009).

Il est intéressant de noter que, alors que l'avance technologique est due à une asymétrie temporelle (diachronique) dans la détention de compétences entre des organisations, la modularité est le fait d'une asymétrie spatiale (synchronique) dans la détention de compétences au sein des organisations. Néanmoins, ces pratiques suivent le même principe d'action : elles visent toutes les deux à prévenir l'acquisition de compétences en matière d'imitation par un acteur donné au travers de l'établissement d'un écart de compétences avec le détenteur de l'innovation.

\section{Conclusion}

Jusqu'à présent, dans la littérature académique, les différents mécanismes de protection des

2 Ces deux remarques sont importantes dans la mesure où elles s'opposent à une vision courante selon laquelle la nature des connaissances et l'intensité compétitive d'une industrie constitueraient en eux-mêmes des mécanismes de protection. Nous rejoignons d'autres auteurs pour considérer au contraire que ces facteurs jouent un rôle modérateur relativement à l'avance technologique, mais n'offrent pas directement de protection. 
innovations contre l'imitation avaient généralement été listés plutôt que clairement articulés au sein d'un cadre d'analyse cohérent. En conséquence, un inventaire exhaustif de ces mécanismes était manquant. Dans cet article, nous avons donc tenté de traiter ce problème, en proposant un cadre d'analyse qui identifie six mécanismes génériques de protection des innovations (Tableau 1), et en inventoriant la mise en œuvre concrète de ces six mécanismes génériques au niveau inter- comme intra-organisationnel (Tableau 2). Ce faisant, nous avons ainsi mis en évidence une analogie fonctionnelle entre mécanismes à travers les niveaux d'analyse. En d'autres termes, l'article a permis de montrer que les mécanismes qui relèvent de pratiques de management stratégique au niveau inter-organisationnel comme ceux qui découlent de pratiques de gestion des ressources humaines en interne participent d'une seule et même logique.

D'un point de vue scientifique, le cadre d'analyse générique proposé et l'inventaire des mécanismes de protection associé pourraient permettre de structurer la recherche sur les mécanismes de protection des innovations contre l'imitation. D'une part, ils permettent de prendre acte des travaux antérieurs. On peut ainsi noter que, alors que la plupart des mécanismes inter-organisationnels ont été particulièrement étudiés, les normes de propriété intellectuelle au niveau inter-organisationnel ainsi que les mécanismes intra-organisationnels ont fait l'objet de moins d'attention. D'autre part, le cadre d'analyse générique proposé et l'inventaire des mécanismes de protection associé permettent d'identifier des pistes pour de futures recherches. Il serait notamment souhaitable (1) de poursuivre l'étude des normes de protection intellectuelles au niveau inter-organisationnel ainsi que celle des mécanismes intra-organisationnels, (2) d'accentuer les travaux sur les relations de substitution entre mécanismes de protection, qui sont en nombre limité, et enfin, (3) de poursuivre les travaux sur les relations de complémentarité entre mécanismes de protection, qui est un thème encore peu étudié. Par ailleurs, alors que la protection des innovations est un enjeu important pour les économies de la connaissance contemporaines, il n'est pas certain que les inventeurs soient toujours au fait des mécanismes de protection à leur disposition, ce qui est susceptible d'entraîner des détournements 
d'innovations qui pourraient être évités. D'un point de vue managérial, ce cadre d'analyse générique et l'inventaire associé qui expose clairement les différents mécanismes de protection existants pourraient donc également offrir un vade mecum utile aux praticiens. Dans le même temps, ce travail pourrait également venir nourrir les réflexions politiques, en permettant d'identifier les mécanismes socialement désirables parmi ceux qui existent.

\section{Bibliographie}

Baldwin Carliss Y. et Henkel Joachim, 2014, « Modularity and intellectual property protection »,. Strategic Management Journal.

Borgatti Stephen P. et Halgin Daniel S., 2011, « On network theory »,. Organization Science, vol. 22, $\mathrm{n}^{\circ}$ 5, p. 1168-1181.

Conti Raffaele, 2014, « Do non-competition agreements lead firms to pursue risky R\&D projects? »,. Strategic Management Journal, vol. 35, n 8, p. 1230-1248.

Costas Jana et Grey Christopher, 2014, « Bringing Secrecy into the Open: Towards a Theorization of the Social Processes of Organizational Secrecy »,. Organization Studies, vol. 35, n 10, p. $1423-1447$.

Delerue Hélène et Lejeune Albert, 2010, « Job mobility restriction mechanisms and appropriability in organizations: The mediating role of secrecy and lead time »,. Technovation, vol. $30, n^{\circ} 5$, p. 359-366.

Di Stefano Giada, King Andrew A. et Verona Gianmario, 2014, « Kitchen confidential? Norms for the use of transferred knowledge in gourmet cuisine »,. Strategic Management Journal, vol. $35, n^{\circ} 11$, p. $1645-1670$.

Fauchart Emmanuelle et Von Hippel Eric, 2008, « Norms-based intellectual property systems: The case of French chefs »,. Organization Science, vol. 19, n² 2, p. 187-201.

Gallié Emilie-Pauline et Legros Diégo, 2012, « French firms’ strategies for protecting their intellectual property »,. Research Policy, vol. 41, n 4, p. 780-794.

Ganco Martin, Ziedonis Rosemarie H. et Agarwal Rajshree, 2015, « More stars stay, but the brightest ones still leave: Job hopping in the shadow of patent enforcement »,. Strategic Management Journal, vol. 36, n 5, p. 659-685.

Haeussler Carolin, 2011, « Information-sharing in academia and the industry: A comparative study »,. Research Policy, vol. 40, n 1, p. 105-122.

Hagedoorn John, Cloodt Danielle et Van Kranenburg Hans, 2005, « Intellectual property rights and the governance of international R\&D partnerships »,. Journal of International Business Studies, vol. 36, $\mathrm{n}^{\circ}$ 2, p. 175-186. 
Hallen Benjamin L., Katila Riitta et Rosenberger Jeff D., 2014, « How Do Social Defenses Work? A Resource-Dependence Lens on Technology Ventures, Venture Capital Investors, and Corporate Relationships »,. Academy of Management Journal, vol. 57, n 4, p. 1078-1101.

Hamel Gary, 1991, « Competition for competence and interpartner learning within international strategic alliances »,. Strategic management journal, vol. 12, nº S1, p. 83-103.

Hannah David R., 2005, « Should I keep a secret? The effects of trade secret protection procedures on employees’ obligations to protect trade secrets »,. Organization Science, vol. 16, $\mathrm{n}^{\circ}$ 1, p. 71-84.

Hurmelinna-Laukkanen Pia et Puumalainen Kaisu, 2007, « Nature and dynamics of appropriability: strategies for appropriating returns on innovation »,. R\&d Management, vol. 37, $\mathrm{n}^{\circ} 2$, p. 95112.

James Sharon D., Leiblein Michael J. et Lu Shaohua, 2013, « How firms capture value from their innovations »,. Journal of management, vol. 39, n 5, p. 1123-1155.

Kale Prashant, Singh Harbir et Perlmutter Howard, 2000, « Learning and protection of proprietary assets in strategic alliances: building relational capital »,. Strategic Management Journal, vol. 21, n 3, p. 217-237.

Keupp Marcus Matthias, Beckenbauer Angela et Gassmann Oliver, 2009, « How managers protect intellectual property rights in China using de facto strategies »,. R\&d Management, vol. 39, $\mathrm{n}^{\circ} 2$, p. 211-224.

Kim Jinyoung et Marschke Gerald, 2005, « Labor mobility of scientists, technological diffusion, and the firm's patenting decision »,. RAND Journal of Economics, p. 298-317.

Lazega Emmanuel, 2000, « Rule enforcement among peers: a lateral control regime »,. Organization Studies, vol. 21, n 1, p. 193-214.

Leiponen Aija et Byma Justin, 2009, « If you cannot block, you better run: Small firms, cooperative innovation, and appropriation strategies »,. Research Policy, vol. 38, n 9, p. 1478-1488.

Li Dan, Eden Lorraine, Hitt Michael A. et Ireland R. Duane, 2008, « Friends, acquaintances, or strangers? Partner selection in R\&D alliances »,. Academy of Management Journal, vol. 51, $n^{\circ} 2$, p. 315-334.

Liebeskind Julia Porter, 1997, « Keeping organizational secrets: Protective institutional mechanisms and their costs »,. Industrial and Corporate Change, vol. 6, n 3, p. 623-663.

Liebman Kenneth A., Kahnke Randall E. et Bundy Kerry L., 2005, « Inevitable Disclosure: Dealing with Trade Secret Issues When Hiring a New Employee »,. Intellectual Property \& Technology Law Journal, vol. 17, n 2, p. 21-23.

Mandard Matthieu, 2013, «Profiter de l'innovation collaborative : alliances de R\&D et mécanismes de protection des actifs technologiques »,. Management \& Avenir, vol. , n² 2, p. 120-138.

Marx Matt, 2011, « The firm strikes back non-compete agreements and the mobility of technical professionals »,. American Sociological Review, vol. 76, n 5, p. 695-712. 
McEvily Susan K. et Chakravarthy Bala, 2002, « The persistence of knowledge-based advantage: an empirical test for product performance and technological knowledge »,. Strategic management journal, vol. 23, n 4, p. 285-305.

Oliar Dotan et Sprigman Christopher, 2008, « There’s No Free Laugh (Anymore): The Emergence of Intellectual Property Norms and the Transformation of Stand-Up Comedy »,. Virginia Law Review, p. 1787-1867.

Oxley Joanne E, 1999, « Institutional environment and the mechanisms of governance: the impact of intellectual property protection on the structure of inter-firm alliances »,. Journal of Economic Behavior \& Organization, vol. 38, n 3, p. 283-309.

Oxley Joanne E. et Sampson Rachelle C., 2004, « The scope and governance of international R\&D alliances »,. Strategic Management Journal, vol. 25, n 8-9, p. 723-749.

Samuelson Pamela et Scotchmer Suzanne, 2002, « The law and economics of reverse engineering »,. Yale Law Journal, p. 1575-1663.

Somaya Deepak, 2012, « Patent strategy and management: An integrative review and research agenda »,. Journal of Management, vol. 38, n 4, p. 1084-1114.

Teece David J., 1986, « Profiting from technological innovation: Implications for integration, collaboration, licensing and public policy »,. Research Policy, vol. 15, n 6, p. 285-305. 